\title{
Autoregulated expression of the gene coding for the leucine-responsive protein, Lrp, a global regulator in Salmonella enterica serovar Typhimurium
}

Correspondence

Charles J. Dorman

cjdorman@tcd.ie

Received 10 March 2008

Revised 17 April 2008

Accepted 23 April 2008

\author{
Kirsty A. McFarlandt and Charles J. Dorman
} Department of Microbiology, Moyne Institute of Preventive Medicine, Trinity College, Dublin 2,
Ireland

\begin{abstract}
In this study, the Irp gene encoding the leucine-responsive regulatory protein (Lrp) in Salmonella enterica serovar Typhimurium was found to be negatively autoregulated. Its transcription start site was determined by primer extension analysis, showing that the Irp promoter is located at a different site to that inferred previously from the $S$. Typhimurium genome sequence. Chromosomal DNA fragments that include the promoter region were bound by purified Lrp protein in vitro, producing up to four distinct protein-DNA complexes. DNase I footprinting identified regions that were protected by the protein in vitro as well as bases that became hypersensitive to DNase I treatment following Lrp binding. A clear pattern of periodic hypersensitivity was detected between positions -130 and +15 that was consistent with wrapping of the DNA around Lrp in a nucleoprotein complex that includes the putative promoter region. Lrp-DNA interaction in this region was fully consistent with the observed repression of Irp transcription by this protein. Leucine was found to modulate Lrp-mediated autorepression by remodelling the Lrp-DNA nucleoprotein complex.
\end{abstract}

\section{INTRODUCTION}

The Lrp protein is an important global regulator in prokaryotes (Brinkman et al., 2003) and the archaea (Bell \& Jackson, 2000; Brinkman et al., 2002). In Salmonella and Escherichia coli, Lrp is a DNA-binding protein with a molecular mass of $18.8 \mathrm{kDa}$ whose activity can be modulated by L-leucine. This amino acid influences the oligomeric state of Lrp, with octamer formation being favoured in its presence (Chen et al., 2001, 2005; Chen \& Calvo, 2002; De los Rios \& Perona, 2007; Peterson et al., 2007). Lrp can activate or repress gene expression, and depending on the system subject to Lrp regulation, leucine can play a stimulatory, inhibitory or neutral role (Brinkman et al., 2003; Calvo \& Matthews, 1994). Lrp activity is not restricted to transcriptional control: it can affect other DNA transactions, such as site-specific recombination (Kelly et al., 2006; Roesch \& Blomfield, 1998).

Lrp has roles in Salmonella that are distinct from its contributions in E. coli. These include regulating the expression of the $s p v$ virulence genes on the Salmonella

tPresent address: Division of Infectious Diseases, Children's Hospital, Enders Building, Harvard University, 300 Longwood Avenue, Boston, MA 02115, USA.

Abbreviation: EMSA, electrophoretic mobility shift assay. virulence plasmid (Marshall et al., 1999), which are important for the establishment of a systemic infection in the host (Libby et al., 2000; Rotger \& Casadesús, 1999; Paesold et al., 2002). Lrp is also a regulator of the pef fimbrial genes on the same plasmid (Nicholson \& Low, 2000). The mechanism by which Lrp regulates pef genes involves competition with the DNA adenine methylase for access to key $5^{\prime}$-GATC-3' sites in the pef regulatory region that determine the expression state of the fimbrial operon (Nicholson \& Low, 2000). This mechanism is similar to the one that controls expression of the pap fimbrial genes in uropathogenic strains of E. coli (Braaten et al., 1992; Hernday et al., 2002; van der Woude et al., 1992). In addition, Lrp controls the expression of the virulence plasmid traJ gene (Camacho \& Casadesús, 2005), allowing Lrp to influence the conjugal transfer of this episome (Camacho \& Casadesús, 2002). On the Salmonella chromosome, the Lrp protein regulates type 1 fimbrial gene expression through a mechanism that is completely distinct from that used to control type 1 fimbrial genes in E. coli (Kelly et al., 2006; McCusker et al., 2008; McFarland et al., 2008; Roesch \& Blomfield, 1998). Instead of influencing the efficiency of a site-specific recombination process that moves the E. coli fim genes between ON and OFF expression states, Lrp in Salmonella enterica controls fim gene expression by governing the supply of a regulatory protein, FimZ (McFarland et al., 2008). 
Despite its involvement in many important regulatory processes, the $S$. enterica $\operatorname{lr} p$ gene has not been previously characterized. Bioinformatic analysis of the S. enterica LT2 genome sequence has suggested locations for the $\operatorname{lrp}$ transcription start signals; however, the inferred binding sites for RNA polymerase differ from the consensus sequences for promoters used by RNA polymerase holoenzyme containing sigma-70 or any other Salmonella sigma factor (McClelland et al., 2001). In this study we cloned the $\operatorname{lrp}$ gene from the $S$. enterica serovar Typhimurium mouse-virulent strain SL1344 and characterized its regulation at the level of transcription using both in vivo and in vitro methods. The transcription control elements were found to be in a different location from that suggested by the available bioinformatic information. The $S$. enterica lrp gene was found to share several regulatory features with its well-characterized counterpart in E. coli K12 , although there were also some features that were specific to $S$. enterica.

\section{METHODS}

Growth conditions. Bacteria were cultured at $37{ }^{\circ} \mathrm{C}$ with shaking at 250 r.p.m., either in LB broth or in MOPS minimal broth $(0.4 \%$, w/v, glucose, $0.5 \mathrm{mM}$ histidine) which was supplemented with $10 \mathrm{mM} \mathrm{L}$ leucine when required. Overnight cultures of bacteria in $3 \mathrm{ml} \mathrm{LB}$ broth were either inoculated by $1: 100$ dilution into $25 \mathrm{ml}$ fresh LB broth, or washed twice with warmed MOPS minimal broth before inoculation $(1: 50)$ into $25 \mathrm{ml}$ MOPS minimal broth. Optical densities of bacterial suspensions were measured at $600 \mathrm{~nm}$ and equal numbers of bacteria were used for inoculation.

Strains, plasmids and oligonucleotides. The strains and plasmids are described in Table 1 and the oligonucleotides used in this study are listed in Table 2. Plasmid pZEPlrp was produced by PCR amplification of a $612 \mathrm{bp}$ fragment, including $61 \mathrm{bp}$ of the $\operatorname{lrp}$ ORF and $533 \mathrm{bp}$ of the $\operatorname{lr} p$ regulatory region of SL1344, using primer pair lrp-gfp-F and lrp-gfp-R (Table 1). This fragment and the parental vector pZEP08 were each digested with $S m a \mathrm{I}$ and $\mathrm{XbaI}$, purified and ligated. The plasmid pBSK.lrp was used for DNase I footprinting and associated DNA sequencing reactions. It was generated by PCR of SL1344 DNA with primer pair $l r p$-F.BamHI and $l r p$-R.EcoRI, followed by cloning into the multi-cloning site of pBluescript II SK (-). Plasmid pKMC101 was used to determine the $\operatorname{lrp}$ transcription start site by primer extension analysis. This plasmid was produced by PCR amplification of the $\operatorname{lrp}$ gene and its flanking regions using primer pair Fwd1.3-EcoRI and Rev1.3-PstI followed by restriction enzyme digestion and ligation of the resulting DNA fragment into the EcoRI and PstI sites in pCL1921.

Flow cytometry. Bacterial samples were fixed by dilution to

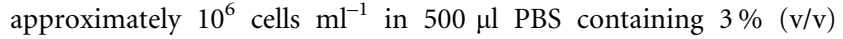
formaldehyde. The samples were examined for GFP expression using the EPICS-XL flow cytometer (Beckman Coulter). Assays were performed in triplicate and mean values are reported.

Electrophoretic mobility shift assay (EMSA). DNA probes were amplified using biotinylated primers to the region of interest (Table 2) and were subsequently purified by gel electrophoresis. Approximately 100 pg biotinylated DNA probe was incubated with binding buffer for $5 \mathrm{~min}$ at room temperature as described by Camacho \& Casadesús (2002). Increasing concentrations of Lrp protein were added, followed by incubation for $20 \mathrm{~min}$ at room temperature. Protein-DNA complexes were formed in a reaction volume of $20 \mu \mathrm{l}$, including leucine where applicable. A $10 \mu \mathrm{l}$ aliquot of the protein-DNA mixture, plus loading dye, was subjected to electrophoresis at $100 \mathrm{~V}$ on Novex 6\% DNA Retardation gels (Invitrogen) at room temperature. Gels were transferred onto membrane using the Novex XCell II blot module (Invitrogen), UV-cross-linked, and developed using the Chemiluminescent Nucleic Acid Detection Module (Pierce).

DNase I footprinting. DNase I footprinting was carried out as previously described (McFarland et al., 2008). The probes used for DNase I footprinting were generated by PCR using primer pairs lrpF.BamHI and $l r p$-R.EcoRI (Table 2) with pBSK.lrp as the DNA template. The sequences complementary to the T7 oligonucleotides are located externally to the multi-cloning site of pBluescript II SK $(-)$. T4 polynucleotide kinase was used to label the DNA probes at both ends with $\left[\gamma_{-}{ }^{32} \mathrm{P}\right] \mathrm{ATP}$, which were then digested to remove the label from one end, depending on the strand to be analysed. Lrp protein-DNA binding reactions used for DNase I footprinting were identical to those used for EMSA analysis, except that these reactions were performed in $50 \mu \mathrm{l}$ volumes. DNA sequencing reactions using dideoxy chain-terminators were performed as previously described

Table 1. Bacterial strains and plasmids

\begin{tabular}{|c|c|c|}
\hline Strain or plasmid & Relevant characteristics & Source \\
\hline \multicolumn{3}{|l|}{ Strains } \\
\hline SL1344 & rpsL hisG & Hoiseth \& Stocker (1981) \\
\hline CJD3130 & SL1344 lrp:: kan & McFarland et al. (2008) \\
\hline CJD3132 & SL1344 pZEPlrp & This study \\
\hline CJD3133 & CJD3130 pZEPlrp & This study \\
\hline \multicolumn{3}{|l|}{ Plasmids } \\
\hline pBluescript II SK (-) & Cloning vector, $\mathrm{Amp}^{\mathrm{R}}$ & Stratagene \\
\hline pBSK.lrp & $418 \mathrm{bp} \operatorname{lrp}$ promoter in pBluescript II SK & This study \\
\hline pZEP08 & Promoterless $g f p, \mathrm{Cml}^{\mathrm{R}} \mathrm{Amp}^{\mathrm{R}}$ & Hautefort et al. (2003) \\
\hline pZEPlrp & $\begin{array}{l}612 \text { bp } \operatorname{lr} p \text { promoter region upstream } \\
\text { of promoterless } g f p \text { in pZEP08 }\end{array}$ & This study \\
\hline pCL1921 & pSC101 replicon, $\operatorname{Spc}^{\mathrm{R}} \operatorname{Str}^{\mathrm{R}}$ & Lerner \& Inouye (1990) \\
\hline pKMC101 & $\begin{array}{l}1.3 \mathrm{~kb} \operatorname{lrp} \text { gene and flanking regions } \\
\text { cloned into pCL1921 }\end{array}$ & This study \\
\hline
\end{tabular}


Table 2. Oligonucleotides

\begin{tabular}{|ll|}
\hline Name & \multicolumn{1}{c|}{ Sequence $\left(\mathbf{5}^{\prime}-\mathbf{3}^{\prime}\right)^{*}$} \\
\hline Fwd $1.3-$-EcoRI & ATAGAATTCGTTGTTGGCAGACAATGAGCAGAATTGTAGG \\
Rev $1.3-$ - $s t \mathrm{I}$ & ATACTGCAGGGGCAATAAGTATCAACAACGCTTCCAAAAG \\
$l r p-g f p-\mathrm{F}$ & ATACCCGGGGTTGTTGGCAGACAATG \\
$l r p-g f p-\mathrm{R}$ & CGCTCTAGATAAGAATGTTACGATCG \\
PE- $l p p$ & GAATGTTACGATCGATACGGTCGAGATCTTTG \\
$l r p$-F. B $a m \mathrm{H} \mathrm{HI}$ & ATCGGATCCGTTGTTGGCAGACAATGAG \\
$l r p$-R.EcoRI & GCGGATTCGCCTGGTCTATGGTGAGAAG \\
$l r p$-EMSA-F & GTTGTTGGCAGACAATGAGC \\
$l r p$-EMSA-F3 & TGTAATACCATGTTTACCGG \\
$l r p$-EMSA-F4 & ATCGATGTTTTGCTTTGAC \\
$l r p$-EMSA-F5 & GACAGCGACGTTATCATCAC \\
$l r p$-R.Bio & GCCTGGTCTATGGTGAGAAG \\
$l r p$-EMSA-R3 & GTTCATCGGTTCATGCTATTAC \\
$l r p$-EMSA-R4 & CAGTTATTCATTAGAGAGGGCG \\
$l r p$-EMSA-R5Bio & TACATGCATGATTATGCAGC \\
\hline
\end{tabular}

${ }^{\star}$ Restriction enzyme sequences: GAATTC, EcoRI; CTGCAG, Psti; CCCGGG, SmaI; TCTAGA, XbaI; GGATCC, BamHI.

(McFarland et al., 2008), using the T4 DNA sequencing kit (USB) and appropriate sequencing primers. The products of the DNase I footprinting and the DNA sequencing reactions were resolved by electrophoresis through a $6 \%$ urea-polyacrylamide gel.

Primer extension analysis. RNA was extracted from SL1344 that had been grown to $\mathrm{OD}_{600} 3.5$ in LB broth (approx. 5.5 h growth), and to $\mathrm{OD}_{600} 0.5$ in MOPS minimal broth, using the SV Total RNA isolation kit (Promega). The oligonucleotide PE- $\operatorname{lrp}$ (Table 2) is complementary to $32 \mathrm{bp}$ of the $\operatorname{lrp}$ mRNA, $58 \mathrm{nt}$ into the ORF. PE-lrp was labelled with $\left[\gamma_{-}{ }^{32} \mathrm{P}\right] \mathrm{ATP}$ using T4 phosphonucleotide kinase (New England Biolabs) according to the manufacturer's instructions. The SuperScript III Reverse Transcriptase kit (Invitrogen) was used to perform the primer extension reaction; the product was then purified by ethanol precipitation, resuspended in formamide loading dye, and heated to $95{ }^{\circ} \mathrm{C}$ for $10 \mathrm{~min}$. Samples were analysed by electrophoresis on denaturing $6 \%$ polyacrylamide gels alongside sequencing reactions that were generated using primer PE-lrp and pKMC101 as template.

\section{RESULTS}

\section{Loss of Lrp expression has a modest negative effect on the growth of strain SL1344}

The creation of the $\operatorname{lrp}$ knockout mutant of $S$. Typhimurium strain SL1344 has been described previously (McFarland et al., 2008). It was important to assess the impact of the lrp mutation on the physiology of the bacterium. For this reason, the growth of the lrp knockout mutant was compared with the wild-type in the complex medium LB and in MOPS minimal medium (with and without leucine) over a $24 \mathrm{~h}$ period. In LB, the growth curves of the wild-type and mutant were essentially superimposable, although the wild-type had a slightly shorter doubling time $(28 \mathrm{~min})$ than the $\operatorname{lrp}$ mutant (30 min). In MOPS minimal medium, the mutant had a doubling time that was $\sim 30 \%$ longer than that of the wildtype during the exponential phase of growth; the two strains achieved approximately similar culture densities in stationary phase. This pattern was mildly affected by the addition of leucine: there was some reduction in the growth of the wild-type in exponential phase and the $\operatorname{lrp}$ mutant had a shortened lag phase. These data implied an important role for Lrp in the physiology of $S$. Typhimurium growing in minimal medium. They also showed that, in contrast to the situation in E. coli (Bouloc et al., 1992), leucine did not abolish the growth of the bacteria in minimal medium. The reason for this is not known.

\section{Negative autoregulation of Irp transcription}

The putative $\operatorname{lr} p$ transcription signals were cloned into the plasmid pZEP08 to create an $l r p-g f p$ transcriptional fusion (Methods). This plasmid, pZEPlrp, allowed the green fluorescent protein to be used as a reporter of $\operatorname{lrp}$ promoter activity. The plasmid was introduced into the SL1344 wildtype and the SL1344 lrp knockout mutant. This allowed the effect of the lrp mutation on the activity of the lrp promoter to be measured. Strains SL1344 and SL1344 $\operatorname{lrp}$ grew with similar growth curves in LB when harbouring the pZEPlrp plasmid (Fig. 1a). In MOPS minimal medium, the $\operatorname{lrp}$ mutant grew more slowly than the wild-type and the presence of leucine had a minor negative influence on growth (Fig. 2a).

The absence of the Lrp protein resulted in increased $\operatorname{lr} p$ promoter activity in cultures growing in LB (Fig. 1b) or in minimal MOPS (Fig. 2b). When leucine was added to the MOPS culture, the level of $l r p-g f p$ expression increased by between 1.5- and 2-fold, provided the Lrp protein was present; leucine did not have this effect in the lrp knockout mutant (Fig. 2b). These data showed that Lrp repressed the transcription of the $\operatorname{lr} p$ gene and that this negative effect was partially alleviated by the presence of leucine. 

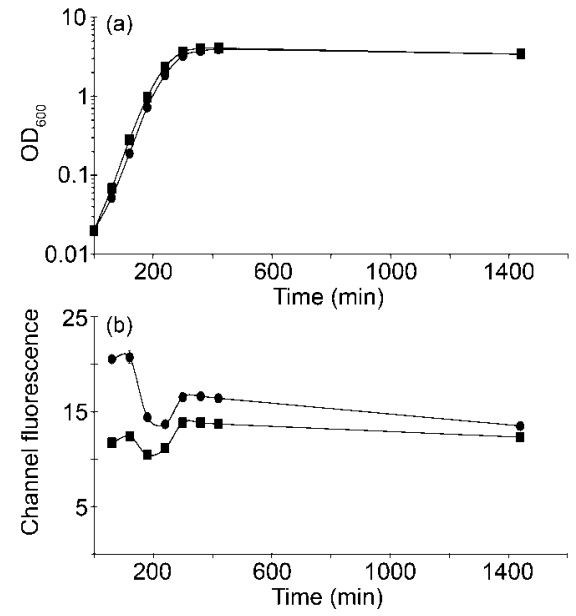

Fig. 1. Expression of an Irp-gfp transcriptional fusion in the presence and absence of Lrp in LB. (a) Growth curves of the Irp-

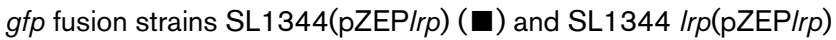
(0) in LB. (b) Gfp expression from Irp-gfp in the wild-type ( $\boldsymbol{\square})$ and the Irp mutant (O) measured by flow cytometry. The experiment was performed in triplicate; means \pm SE are plotted (not shown where smaller than symbols).

\section{Identifying the Irp transcription start site}

It was necessary to identify the transcription start site of the $S$. Typhimurium $\operatorname{lr} p$ gene by molecular methods. Previous estimates based on bioinformatic techniques suggested that the lrp promoter had several non-standard features, in particular very poor matches to the -35 and -10

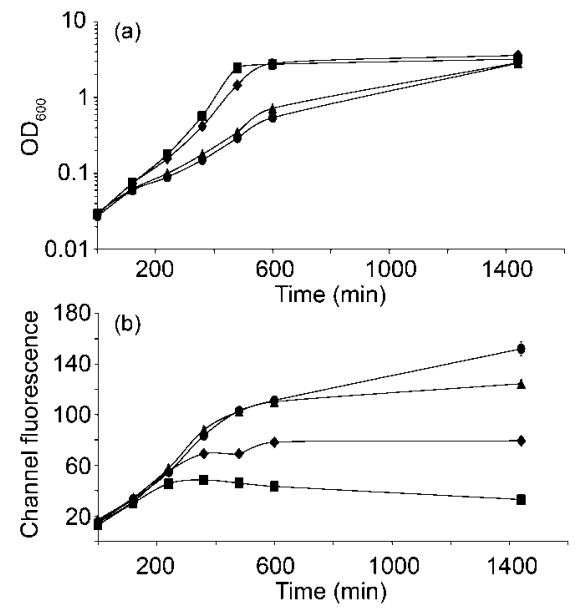

Fig. 2. Expression of an Irp-gfp transcriptional fusion in the presence and absence of leucine in MOPS minimal medium. (a) Growth curves of the wild-type grown with $(\boldsymbol{)})$ or without $(\boldsymbol{\square})$ leucine and the Irp mutant grown with $(\boldsymbol{\Lambda})$ or without $(\boldsymbol{\bullet})$ leucine. (b) Gfp expression from Irp-gfp in the wild-type grown with ( $)$ or without $(\boldsymbol{\square})$ leucine and in the Irp mutant grown with $(\boldsymbol{\Delta})$ or without (O) leucine. hexanucleotide motifs that are bound by the RpoD sigma factor of RNA polymerase (McClelland et al., 2001). If these were confirmed, they would have to be taken into account in assembling a model of $\operatorname{lrp}$ gene regulation.

The transcription start site was determined using a standard primer extension procedure (Methods) and mapped to a cluster of A bases located $257 \mathrm{bp}$ upstream from the translation start codon of the lrp ORF (Fig. 3). The start site was 10 bases further downstream of the position described previously for the $\operatorname{lrp}$ gene in E. coli K12 (Wang et al., 1994). Moreover, the likely binding sites for RNA polymerase were poor matches to the standard features associated with sigma-70 promoters, in keeping with the weak nature of the promoter. The -35 hexamer matched the consensus sequence in three out of six positions and the -10 had only two matches out of six to the consensus (Fig. 3). These data showed that the $S$. Typhimurium lrp transcription start site determined previously using in silico methods was likely to be incorrect.

The primer extension experiment was performed using mRNA extracted from two SL1344 cultures, one grown in LB and one grown in MOPS minimal medium. The intensity of the extension product showed that the gene was transcribed to a higher level in the culture growing in the minimal medium (Fig. 3), in agreement with the data obtained using the $\operatorname{lrp}$-gfp reporter fusion (Figs $2 \mathrm{~b}$ and $3 \mathrm{~b}$ ).

\section{Purified Lrp binds to the Irp promoter region}

Purified Lrp protein (McFarland et al., 2008) was used in a series of EMSAs to assess Lrp interaction with the transcriptional regulatory region of the SL1344 $\operatorname{lrp}$ gene (Fig. 4). Experiments were performed initially with a DNA fragment that extended from $115 \mathrm{bp}$ upstream of the translation initiation codon to 418 bp further upstream, spanning from -275 to +143 with respect to the transcription start site (Fig. 4a). The labelled DNA fragment was incubated with Lrp protein at a range of concentrations, and parallel incubations were performed in the presence of leucine or glycine. Two Lrp-DNA complexes were formed in all three cases (Fig. 4b). Leucine encouraged the formation of the slowest-migrating Lrp-DNA complex at the expense of the faster-migrating one; glycine did not have this effect. These results showed that Lrp could bind to the lrp regulatory region in the absence of amino acids and that leucine could modulate this process.

The 418 bp DNA fragment was subdivided into six shorter sections that were numbered according to length. $F$ probes had a common end that was distal to the lrp open reading frame while $\mathrm{R}$ probes had a common end that was proximal to it; the fragments were numbered according to their lengths in bp (Fig. 4a). The six DNA fragments, F102, F200, F311, R111, R202 and R310, were used in EMSAs with purified Lrp protein. Only F311 and R310 formed complexes with the protein (Fig. 4c). These two fragments 
(a)

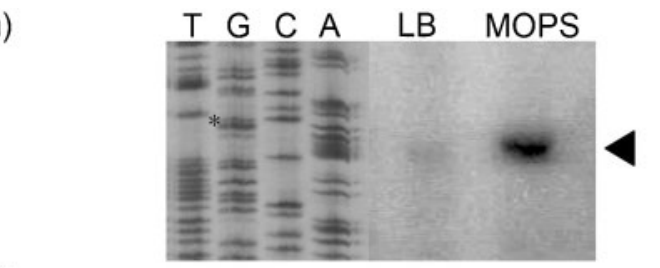

(b)

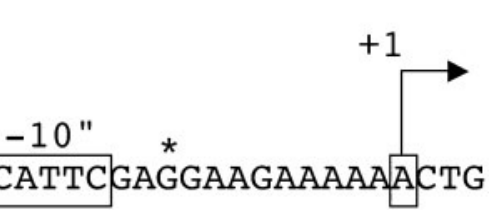

Fig. 3. Determining the location of the Irp transcription start site. The Irp transcription start site was located by primer extension of Irp mRNA. The products of primer extension reactions were electrophoresed next to a dideoxy DNA sequencing ladder generated using the same primer. The RNA used in the primer extension experiments was isolated from bacteria growing in LB or MOPS minimal medium; the Irp promoter was much more active in the latter. (a) A composite of two exposures of the same gel in which an overexposed version of the extension products is shown to allow the bands to be seen clearly. The filled arrowhead indicates the Irp transcript. (b) Sequence of the $S$. Typhimurium Irp regulatory region. The angled arrow labelled ' +1 ' indicates the transcription start site as determined in (a). The asterisk indicates the position of the start site in $E$. coli (Wang et al., 1994). The boxed sequence motifs represent the putative -10 and -35 hexamers of the $S$. Typhimurium Irp promoter.

shared a common 203 bp stretch of DNA that included the lrp promoter. This region of DNA was amplified and used in a further EMSA with Lrp. Three Lrp-DNA complexes were detected, with a fourth being visible at the highest concentration of protein (Fig. 4d). The formation of the slowest-migrating Lrp-DNA complexes was unaffected by the presence of leucine or glycine.

Taken together, the data from the EMSAs showed that Lrp bound to DNA sequences located between positions -167 and +36 , numbered with respect to the transcription start site $(+1)$ of the $S$. Typhimurium $\operatorname{lrp}$ gene. DNase I footprinting was used to examine Lrp-DNA interaction in this region in more detail. The $418 \mathrm{bp}$ DNA fragment (Fig. $4 \mathrm{a}, \mathrm{b}$ ) was labelled at one end with ${ }^{32} \mathrm{P}$, incubated with increasing concentrations of Lrp protein, and then treated with DNase I. The samples were then separated by electrophoresis on a DNA sequencing gel (Fig. 5). Increasing concentrations of Lrp protein were accompanied by a pattern of protection from, and periodic hypersensitivity to, digestion by DNase I on both the coding (Fig. 5a) and the non-coding DNA strands (Fig. 5b) over a region that extended from -135 to +15 . The region of protection included the $r p$ promoter, a finding that was consistent with its repression by the Lrp protein. There was an abrupt transition between DNase I sensitivity and protection and vice versa between 50 and $100 \mathrm{nM}$ Lrp. The pattern of protection and hypersensitivity to DNase I was subtly altered by the presence and absence of $15 \mathrm{mM}$ leucine. Addition of the amino acid was accompanied by hypersensitivity to DNase I in the promoter region ( -40 to +1 ) on the coding and non-coding strands, a region that was protected by Lrp in the absence of leucine (Fig. 5a, b). Leucine also reversed the sensitivity of bases to DNase I in the regions -90 to -100 and -120 to -130 (Fig. 5a). Several matches to the degenerate consensus sequence for Lrp binding sites (Wang et al., 1994) were identified in the region between -110 and +15 ; these are summarized in Fig. 6 . In several cases, these sequences were flanked by ' $T$ ' residues that had shown hypersensitivity to DNase I digestion in the presence of Lrp.

\section{DISCUSSION}

The knockout mutation in the $\operatorname{lrp}$ gene of $S$. Typhimurium SL1344 altered the growth rate of the bacterium in MOPS minimal medium. The wild-type had a doubling time of $58 \mathrm{~min}$ while the $\operatorname{lrp}$ mutant doubled every $76 \mathrm{~min}$. These growth rates were very similar to those reported previously for E. coli (Tuan et al., 1990).

The $\operatorname{lrp}$ promoter in E. coli has been described as leucinesensitive (Borst et al., 1996) and leucine-insensitive (Lin et al., 1992; Wang et al., 1994). In our study, the lrp promoter in $S$. Typhimurium consistently displayed between a 1.5and 2-fold higher activity in the presence of leucine in bacteria growing in minimal MOPS medium (Fig. 1). This modest positive effect of leucine on $\operatorname{lrp}$ transcription was contingent on the presence of the Lrp protein; the lrp knockout mutant expressed the lrp-gfp fusion to the same level in the presence or the absence of leucine (Fig. 1). We noted a dip in $\operatorname{lrp}$ transcription at the end of exponential growth (Fig. 1b). While we cannot explain this in mechanistic terms, a similar effect was noted previously in the case of the E. coli lrp gene (Landgraf et al., 1996).

EMSAs showed that Lrp binds to a 418 bp DNA fragment and that this binding is not abrogated by the addition of leucine (Fig. 4). It has been shown previously that leucine does not alter the binding of Lrp to the regulatory region of the E. coli lrp gene (Wang et al., 1994). However, in the case of the $S$. Typhimurium gene, leucine (but not glycine) 
(a)

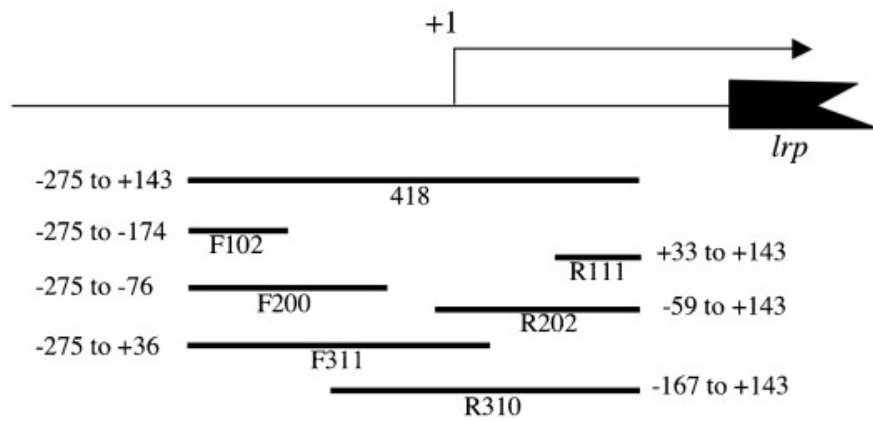

(c)

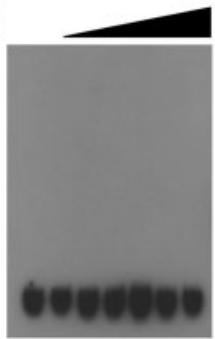

F102

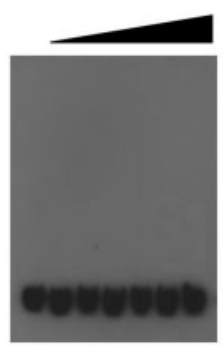

R111

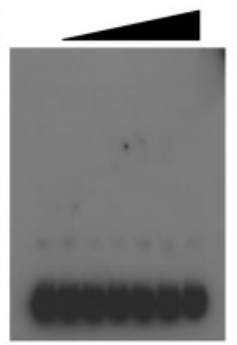

F200

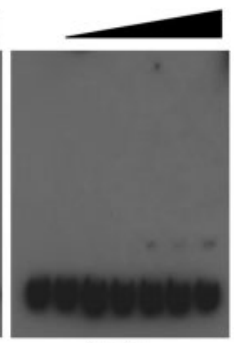

R202

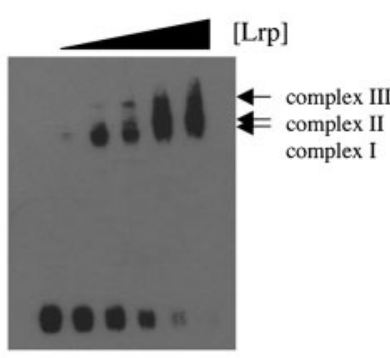

F311

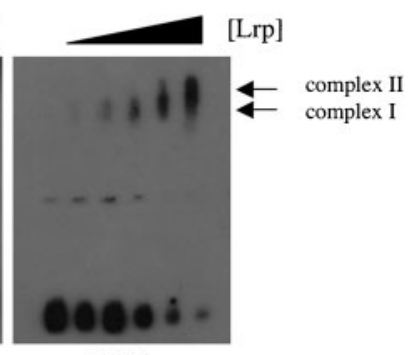

(b)

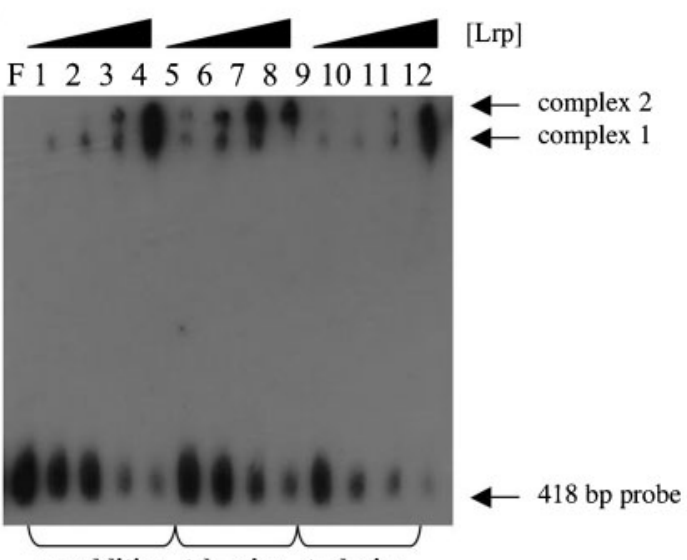

no addition + leucine + glycine

(d)

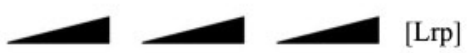

F 12334556789101112

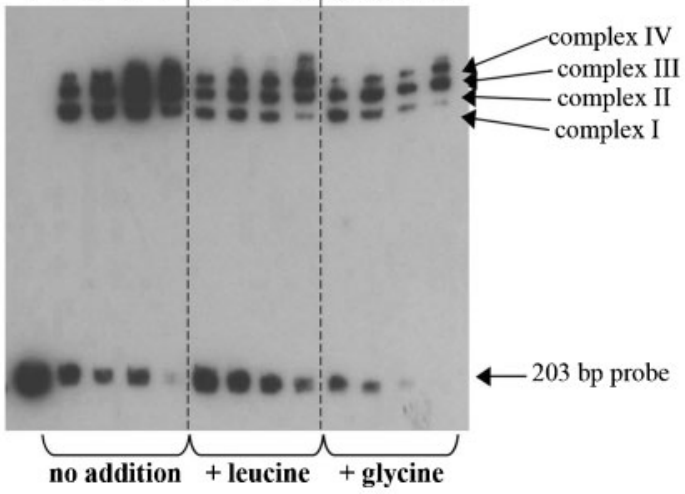

Fig. 4. Binding of the Lrp protein to the Irp regulatory sequences. Binding of the Lrp protein to the Irp gene was examined by EMSA using the purified protein and a series of biotinylated probes derived from a 418 bp DNA fragment that extended from -275 to +143 of the Irp regulatory region. (a) A summary of the DNA probes used in the DNA binding experiments. The horizontal line at the top represents the Irp regulatory region and the angled arrow is the transcription start site, +1 . The $418 \mathrm{bp}$ probe is shown immediately below; its right-hand end abuts $115 \mathrm{bp}$ upstream of the translation start codon of the Irp open reading frame, spanning from -275 to +143 . The three probes coded ' $F$ ' have left ends in common with that of the 418 bp fragment while the three coded ' $R$ ' have common right ends. The numbers in the probe names refer to the lengths of the DNA fragments in base pairs. (b) Interaction of Lrp [13.3 (lane 1), 26.6 (2), 53.2 (3) and 133 (4) nM] with the 418 bp probe in the absence of amino acids or in the presence of $15 \mathrm{mM}$ leucine or glycine. (c) Electrophoretic mobility shift data for each of the three ' $F$ ' and three 'R' probes. Lrp was present at 0, 26.6, 53.2, 106.4, 160, 212.8 and $266 \mathrm{nM}$ for probes F102, R111, F200 and R202. Lrp was present at 0, 13.3, 26.6, 53.2, 133 and $266 \mathrm{nM}$ in the gels with probes F311 and R310. (d) Binding of the Lrp protein [13.3 (lane 1), 26.6 (2), $53.2(3), 133.38$ (4) nM] to a 203 bp DNA fragment with a core sequence that is common to probes R310 and F311. The samples were also incubated with $15 \mathrm{mM}$ leucine or glycine. In each panel, arrows show the positions of the unbound probe and the Lrp-DNA complexes. The first lane in (b) and (d), labelled F, indicates free probe. Experiments were repeated three times and typical results are shown.

alters the relative abundances of the different Lrp-DNA complexes in favour of the slowest-migrating complex. This suggests that leucine changes the nature of the LrpDNA complex without displacing the protein. The details of this alteration to the nucleoprotein complex are unknown but are likely to be subtle given the very modest effect of leucine on $l r p$ promoter activity.
The 418 bp fragment encompasses a 203 bp subfragment that binds Lrp, forming up to four Lrp-DNA complexes in EMSAs (Fig. 4). The number and relative abundances of these complexes do not respond to leucine or glycine. This shows that the subtle leucine-sensitivity of the Lrp-DNA complexes formed with the $418 \mathrm{bp}$ fragment involves DNA sequences not found in this smaller piece of DNA, perhaps 

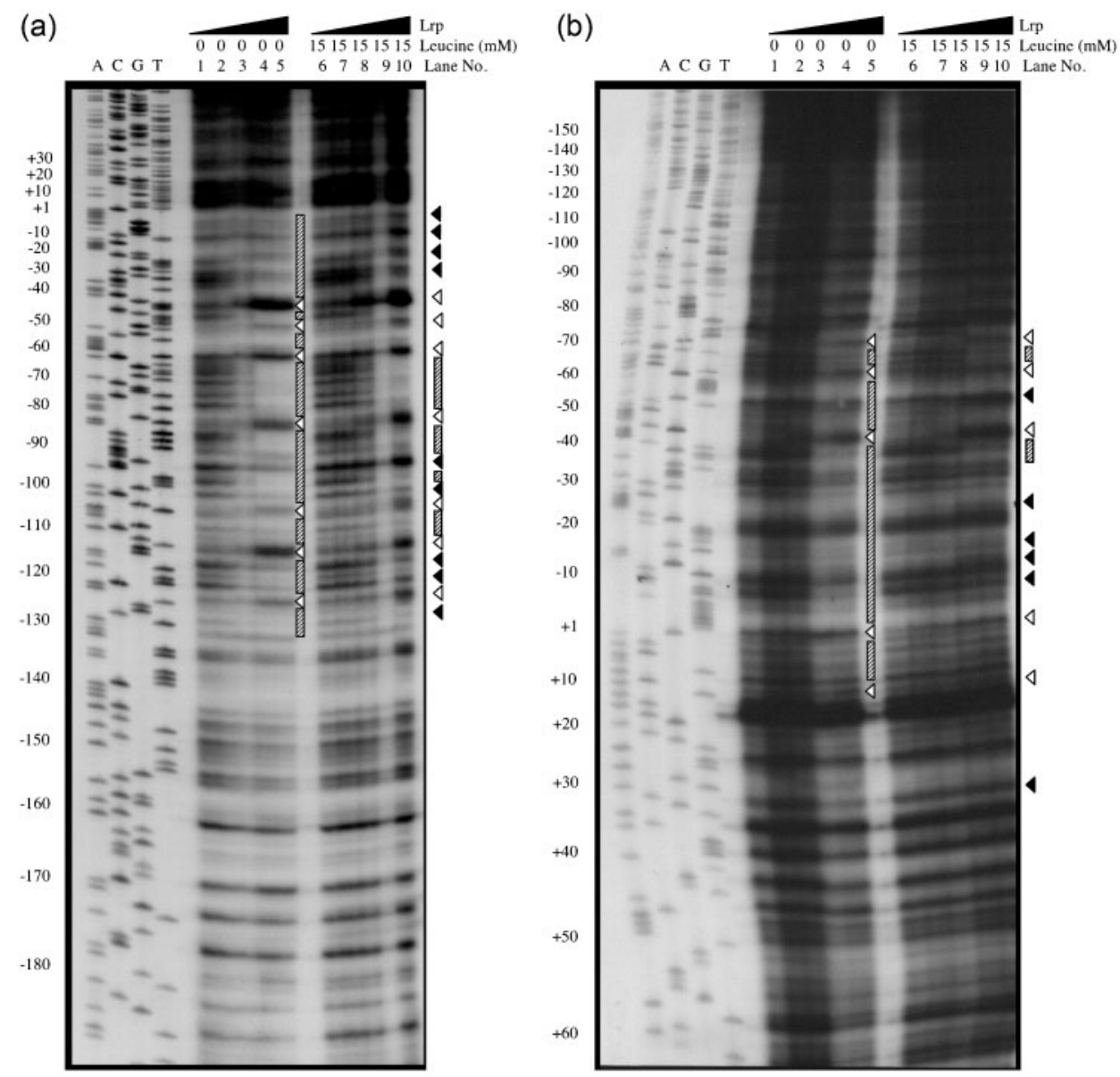

Fig. 5. DNase I analysis of Lrp binding to the Irp regulatory region. DNase I digestion was used to examine the interaction of purified Lrp protein with the coding (a) and non-coding (b) strands of the Irp regulatory region. LRP was added at 0 (lanes 1 and 6), 25 (2 and 7), 50 (3 and 8), 100 (4 and 9) and 150 (5 and 10) $\mathrm{nM}$ in the presence or absence of $15 \mathrm{mM}$ leucine, as indicated. DNase I digestion products were separated on a denaturing polyacrylamide gel next to dideoxy chain-terminator DNA sequencing products (lanes $A, C, G, T$ ) that had been generated using as a sequencing primer the same primer that had been used to make the $203 \mathrm{bp}$ DNA probe. The coordinates of bases in the DNA sequence are shown on the left, numbered with respect to the Irp transcription start site, +1 . Hatched vertical bars indicate regions of the DNA that were protected from DNase I digestion by the Lrp protein; open arrowheads show bases that became hypersensitive to DNase I digestion in the presence of Lrp with and without leucine; filled arrowheads show bases that became hypersensitive to DNase I in the presence of Lrp and leucine. The experiment was repeated on three occasions and a typical result is shown.

due to a requirement for long-range protein-DNA interactions that can only occur on the longest DNA fragments used.

DNase I footprinting reveals Lrp-mediated protection and distortion of the DNA structure between approximately positions -130 and +15 (Fig. 5). Protection is seen most clearly in the absence of leucine on the coding (Fig. 5a, lanes 1 to 5) and non-coding strands (Fig. 5b, lanes 1 to 5). The zones of protection are interrupted by bases showing strong Lrp-mediated hypersensitivity to DNase I. Adding leucine has a subtle effect on the patterns of protection and hypersensitivity. It reduces the overall degree of protection afforded by Lrp and it modifies the pattern of DNase I hypersensitivity. In particular, the protection seen between bases -40 and +1 on the coding strand is replaced by a pattern of periodic hypersensitivity (Fig. $5 \mathrm{a}$ ). This pattern is also detectable on the non-coding strand (Fig. 5b). New regions of hypersensitivity are also seen further upstream on the coding strand, between bases -95 and -130 (Fig. 5a).

The markedly periodic pattern of hypersensitivity to DNase I digestion is consistent with wrapping of the DNA around the Lrp protein, resulting in enhanced exposure of certain bases to DNase I (Nickerson \& Achberger, 1995; Wagner, 2000), and has been reported previously for other DNA sequences that bind Lrp (McFarland et al., 2008; Wang \& Calvo, 1993; Wiese et al., 1997). The 3D structure of the E. coli Lrp protein has been solved (de los Rios \& Perona, 2007). This protein, which is almost identical to Lrp in $S$. 

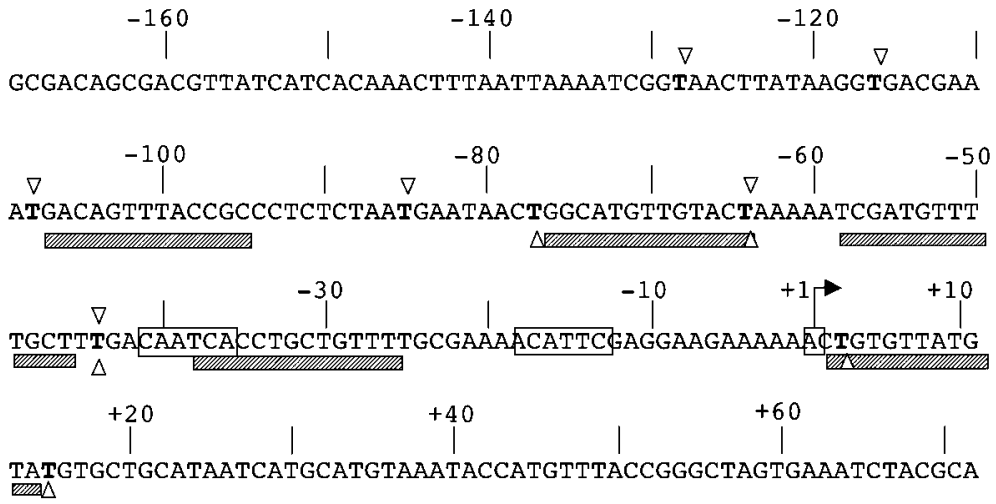

Fig. 6. Locations of Lrp-binding sites at the Irp promoter. Possible matches to the (degenerate) consensus sequence for Lrp-binding sites are underlined by hatched boxes. The coordinates of bases in the sequence are numbered with respect to the Irp transcription start site $(+1)$. The $T$ residues that showed hypersensitivity to DNase I digestion in the presence of Lrp are in bold type. Downward arrowheads show the bases that were hypersensitive on the coding strand; upward arrowheads show those that were hypersensitive on the non-coding strand. Possible -10 and -35 hexameric promoter motifs are boxed.
Typhimurium, can form octamers capable of wrapping approximately $120 \mathrm{bp}$ of DNA, a stretch that is similar in length to that shown here to be affected by Lrp. In the Lrp octamer, the eight helix-turn-helix (H-T-H) DNAbinding motifs located in the amino-terminal domains of each monomer are arranged around the circumference of the octameric structure. This allows the protein to dock with Lrp binding sites distributed along the DNA with a spacing of approximately three helical turns, or $30 \mathrm{bp}$. The fit of each H-T-H motif in the DNA requires adjustment to the Lrp protein structure, suggesting that an induced-fit mechanism operates. It is possible that the binding of leucine to the Regulation of Amino acid Metabolism (RAM) domain in the carboxyl terminus of each Lrp monomer may provide the structural adjustment required to strengthen or to weaken Lrp-DNA interaction, depending on the gene regulatory sequence involved (de los Rios \& Perona, 2007). The modifications to the patterns of protection and hypersensitivity seen in the $\operatorname{lrp}$ regulatory region in the presence and absence of leucine are consistent with an adjustment to the Lrp-DNA contacts due to the influence of the amino acid on Lrp protein structure.

Data from the present study show that Lrp can form up to four complexes with the $S$. Typhimurium lrp promoter region, with one being located downstream of the transcription start site (Fig. 4). DNase I protection data suggest that Lrp binding occurs as far downstream as base +15 , and a leucine-sensitive and DNase-I-hypersensitive base is located at position +35 . This pattern of proteinDNA interaction is consistent with repression of the $\operatorname{lr} p$ promoter by Lrp promoter occlusion. The effect of adding leucine is not to abolish Lrp-DNA interaction, but to remodel it so that the negative influence of the Lrp protein on $l r p$ promoter function is attenuated.

\section{ACKNOWLEDGEMENTS}

This work was supported by a Principal Investigator Programme Grant from Science Foundation Ireland.

\section{REFERENCES}

Bell, S. D. \& Jackson, S. P. (2000). Mechanism of autoregulation by an archaeal transcriptional repressor. J Biol Chem 275, 31624-31629.

Borst, D. W., Blumenthal, R. M. \& Matthews, R. G. (1996). Use of an in vivo titration method to study a global regulator: effect of varying Lrp levels on expression of gltBDF in Escherichia coli. J Bacteriol 178, 6904-6912.

Bouloc, P., Vinella, D. \& D'Ari, R. (1992). Leucine and serine induce mecillinam resistance in Escherichia coli. Mol Gen Genet 235, 242-246.

Braaten, B. A., Platko, J. V., van der Woude, M. W., Simons, B. H., de Graaf, F. K., Calvo, J. M. \& Low, D. A. (1992). Leucine-responsive regulatory protein controls the expression of both pap and fan pili operons in Escherichia coli. Proc Natl Acad Sci U S A 89, 4250-4254.

Brinkman, A. B., Bell, S. D., Lebbink, R. J., de Vos, W. M. \& van der Oost, J. (2002). The Sulfolobus solfataricus Lrp-like protein LysM regulates lysine biosynthesis in response to lysine availability. J Biol Chem 277, 29537-29549.

Brinkman, A. B., Ettema, T. J. G., de Vos, W. M. \& van der Oost, J. (2003). The Lrp family of transcriptional regulators. Mol Microbiol 48, 287-294.

Calvo, J. M. \& Matthews, R. G. (1994). The leucine-responsive regulatory protein, a global regulator of metabolism in Escherichia coli. Microbiol Rev 58, 466-490.

Camacho, E. M. \& Casadesús, J. (2002). Conjugal transfer of the virulence plasmid of Salmonella enterica is regulated by the leucineresponsive regulatory protein and DNA adenine methylation. $\mathrm{Mol}$ Microbiol 44, 1589-1598.

Camacho, E. M. \& Casadesús, J. (2005). Regulation of traJ transcription in the Salmonella virulence plasmid by strand-specific DNA adenine hemimethylation. Mol Microbiol 57, 1700-1718.

Chen, S. \& Calvo, J. M. (2002). Leucine-induced dissociation of Escherichia coli Lrp hexadecamers to octamers. J Mol Biol 318, 1031-1042.

Chen, S., Rosner, M. H. \& Calvo, J. M. (2001). Leucine-regulated selfassociation of leucine-responsive regulatory protein (Lrp) from Escherichia coli. J Mol Biol 312, 625-635.

Chen, S., lannolo, M. \& Calvo, J. M. (2005). Cooperative binding of the leucine-responsive regulatory protein (Lrp) to DNA. J Mol Biol 345, 251-264.

de los Rios, S. \& Perona, J. J. (2007). Structure of the Escherichia coli leucine-responsive regulatory protein (Lrp) reveals a novel octameric assembly. J Mol Biol 366, 1589-1602. 
Hautefort, I., Proenca, M. J. \& Hinton, J. C. D. (2003). Single-copy green fluorescent protein gene fusions allow accurate measurement of Salmonella gene expression in vitro and during infection of mammalian cells. Appl Environ Microbiol 69, 7480-7491.

Hernday, A., Krabbe, M., Braaten, B. \& Low, D. (2002). Selfperpetuating epigenetic pili switches in bacteria. Proc Natl Acad Sci U S A 99, 16470-16476.

Hoiseth, S. K. \& Stocker, B. A. (1981). Aromatic-dependent Salmonella typhimurium are non-virulent and effective as live vaccines. Nature 291, 238-239.

Kelly, A., Conway, C., Cróinin, T. O., Smith, S. G. J. \& Dorman, C. J. (2006). DNA supercoiling and the Lrp protein determine the directionality of fim switch DNA inversion in Escherichia coli K-12. $J$ Bacteriol 188, 5356-5363.

Landgraf, J. R., Wu, J. \& Calvo, J. (1996). Effects of nutrition and growth rate on Lrp levels in Escherichia coli. J Bacteriol 178, 6930-6936.

Lerner, C. G. \& Inouye, M. (1990). Low copy number plasmids for regulated low-level expression of cloned genes in Escherichia coli with blue/white insert screening capability. Nucleic Acids Res 18, 4631.

Libby, S. J., Lesnick, M., Hasegawa, P., Weidenhammer, E. \& Guiney, D. G. (2000). The Salmonella virulence plasmid $s p v$ genes are required for cytopathology in human monocyte-derived macrophages. Cell Microbiol 2, 49-58.

Lin, R., D’Ari, R. \& Newman, E. B. (1992). $\lambda$ placMu insertions in genes of the leucine regulon: extension of the regulon to genes not regulated by leucine. J Bacteriol 174, 1948-1955.

Marshall, D. G., Sheehan, B. J. \& Dorman, C. J. (1999). A role for the leucine-responsive regulatory protein and integration host factor in the regulation of the Salmonella plasmid virulence ( $s p v)$ locus in Salmonella typhimurium. Mol Microbiol 34, 134-145.

McClelland, M., Sanderson, K. E., Spieth, J., Clifton, S. W., Latreille, P., Courtney, L., Porwollik, S., Ali, J., Dante, M. \& other authors (2001). Complete genome sequence of Salmonella enterica serovar Typhimurium LT2. Nature 413, 852-856.

McCusker, M. P., Turner, E. C. \& Dorman, C. J. (2008). DNA sequence heterogeneity in Fim tyrosine-integrase recombinase-binding elements and functional motif asymmetries determine the directionality of the fim genetic switch in Escherichia coli K-12. Mol Microbiol 67, 171-187.

McFarland, K. A., Lucchini, S., Hinton, J. C. D. \& Dorman, C. J. (2008). The leucine-responsive regulatory protein, Lrp, activates transcription of the fim operon in Salmonella enterica serovar Typhimurium via the fimZ regulatory gene. J Bacteriol 190, 602-612.

Nicholson, B. \& Low, D. (2000). DNA methylation-dependent regulation of pef expression in Salmonella typhimurium. Mol Microbiol 35, 728-742.

Nickerson, C. A. \& Achberger, E. C. (1995). Role of curved DNA in binding of Escherichia coli RNA polymerase to promoters. J Bacteriol 177, 5756-5761.

Paesold, G., Guiney, D. G., Eckmann, L. \& Kagnoff, M. F. (2002). Genes in the Salmonella pathogenicity island 2 and the Salmonella virulence plasmid are essential for Salmonella-induced apoptosis in intestinal epithelial cells. Cell Microbiol 4, 771-781.

Peterson, S. N., Dahlquist, F. W. \& Reich, N. O. (2007). The role of high affinity non-specific DNA binding by Lrp in transcriptional regulation and DNA organization. J Mol Biol 369, 1307-1317.

Roesch, P. L. \& Blomfield, I. C. (1998). Leucine alters the interaction of the leucine-responsive regulatory protein (Lrp) with the fim switch to stimulate site-specific recombination in Escherichia coli. $\mathrm{Mol}$ Microbiol 27, 751-761.

Rotger, R. \& Casadesús, J. (1999). The virulence plasmids of Salmonella. Int Microbiol 2, 177-184.

Tuan, L. R., D'Ari, R. \& Newman, E. B. (1990). The leucine regulon of Escherichia coli K-12: a mutation in rblA alters expression of Lleucine-dependent metabolic operons. J Bacteriol 172, 4529-4535.

van der Woude, M. W., Braaten, B. A. \& Low, D. A. (1992). Evidence for global regulatory control of pilus expression in Escherichia coli by Lrp and DNA methylation: model building based on analysis of pap. Mol Microbiol 6, 2429-2435.

Wagner, R. (2000). Transcription Regulation in Prokaryotes. Oxford, UK: Oxford University Press.

Wang, Q. \& Calvo, J. M. (1993). Lrp, a major regulatory protein in Escherichia coli, bends DNA and can organize the assembly of a higher-order nucleoprotein structure. EMBO J 12, 2495-2501.

Wang, Q., Wu, J., Friedberg, D., Platko, J. \& Calvo, J. M. (1994). Regulation of the Escherichia coli lrp gene. J Bacteriol 176, 1831-1839.

Wiese, D. E., Jr, Ernsting, B. R., Blumenthal, R. M. \& Matthews, R. G. (1997). A nucleoprotein activation complex between the leucineresponsive regulatory protein and DNA upstream of the gltBDF operon in Escherichia coli. J Mol Biol 270, 152-168.

Edited by: L. S. Frost 